REVESCO. Revista de Estudios Cooperativos

ISSN: $1885-8031$

https://dx.doi.org/10.5209/REVE.73862

\title{
Emprendimiento social en la formación profesional: el caso del País Vasco
}

\author{
José Luis Monzón Campos ${ }^{1} @$ y Jorge Antonio Torres-Ortega ${ }^{2} @$
}

Recibido: 10 de junio de 2020 / Aceptado: 21 de octubre de 2020 / Publicado: 16 de febrero de 2021

Resumen. Las empresas con fines sociales o empresas de la Economía Social han sido visualizadas como una respuesta innovadora, dinámica, inclusiva y autosostenible a los principales desafíos sociales, económicos y medioambientales que enfrentan nuestras sociedades en la actualidad. Es precisamente en virtud del relevante impacto social y económico que generan las iniciativas empresariales en el ámbito del emprendimiento social que apoyar el surgimiento de emprendedores sociales se ha convertido en una necesidad, especialmente desde edades tempranas. De aquí la importancia de generar datos que permitan ya sea crear o mejorar programas ya existentes de formación de emprendedores, que contemplen entre sus objetivos la formación específica en emprendimiento social. En esta línea, la presente investigación se plantea como objetivo principal caracterizar y comparar a los estudiantes que participan de un proceso formativo en emprendimiento dirigido a jóvenes entre los 16 y 24 años impartido en los Centros de Formación Profesional de la Comunidad Autónoma del País Vasco, España. Concretamente, se busca identificar los principales atributos personales relacionados con la configuración de intenciones de emprender en estos jóvenes, así como cuáles de estos rasgos se relacionan en mayor medida con intenciones de emprender socialmente, vale decir, con la creación de empresas de la Economía Social. La información de este estudio fue obtenida mediante la aplicación de una encuesta a una muestra de 5.767 estudiantes pertenecientes a distintos centros educativos de Euskadi. Los resultados obtenidos revelan que emprendedores potenciales y no emprendedores, así como también emprendedores potenciales sociales y emprendedores potenciales comerciales, constituyen grupos bastante homogéneos. Ahora bien, el análisis de regresión reveló que existe una variable que aumenta significativamente la probabilidad de emprender socialmente, que es justamente uno de los factores que en mayor medida ha sido relevado como propio del emprendimiento social y la Economía Social: la solidaridad.

Palabras clave: Economía Social; Intención emprendedora; Emprendedor social; Teoría de la personalidad; Nivel escolar.

Claves Econlit: L26; L31; L39.

\section{[en] Social entrepreneurship in vocational training: the case of the Basque Country}

\begin{abstract}
Enterprises with social purposes or enterprises of the Social Economy have been viewed as an innovative, dynamic, inclusive and self-sustaining response to the main social, economic and environmental challenges facing our societies today. It is precisely by virtue of the relevant social and economic impact generated by business initiatives in the field of social entrepreneurship that supporting the emergence of social entrepreneurs has become a necessity, especially from an early age. Hence the importance of generating data that allows either creating or improving existing entrepreneurial training programs, which include specific training in social entrepreneurship among its objectives. In this line, the main objective of this research is to characterize and compare the students who participate in a training process in entrepreneurship aimed at young people between the ages of 16 and 24 taught at the Professional Training Centers of the Autonomous Community of the Basque Country, Spain. Specifically, it seeks to identify the main personal attributes related to the configuration of entrepreneurial intentions in these young people, as well as which of these traits are most related to intentions to undertake social endeavours, that is, the creation of organizations of the Social Economy. The information from this study was obtained by applying a survey to a sample of 5767 students belonging to different educational centers in the Basque Country. The results obtained reveal that potential entrepreneurs and non-entrepreneurs, as well as potential social entrepreneurs and potential commercial entrepreneurs, constitute quite homogeneous groups. However, the regression analysis revealed that there is a variable that significantly increases the probability of social entrepreneurship, which is precisely one of the factors that has been highlighted as a key characteristic of social entrepreneurship and Social Economy: solidarity.
\end{abstract}

Keywords: Social Economy; Entrepreneurial intention; Social entrepreneur; Personality theory; School level.

Sumario. 1. Introducción. 2. La Teoría de la Personalidad como explicación del comportamiento emprendedor. 3. El concepto de emprendimiento social y sus subconceptos. 4. Metodología. 5. Resultados. 6. Conclusiones. 7. Referencias bibliográficas.

\footnotetext{
Universidad de Valencia, España.

Dirección de correo electrónico: j.luis.monzon@uv.es

2 Universidad de Santiago, Chile.

Dirección de correo electrónico: jorge.torres@usach.cl.
} 
Cómo citar. Monzón Campos, J.L.; Torres-Ortega, J.A. (2021) Emprendimiento social en la formación profesional: el caso del País Vasco. REVESCO. Revista de Estudios Cooperativos, vol. 137, e73862. https://dx.doi.org/10.5209/reve.73862.

\section{Introducción}

La creación de nuevas empresas se ha considerado como uno de los factores clave para estimular el desarrollo y crecimiento de los territorios y países, ello a través de la generación de nuevos puestos de trabajo, la intensificación de la competencia en los mercados y el impulso a la innovación (Marulanda, Montoya y Vélez, 2014; Sánchez-García, Ward, Hernández y Florez, 2017; Swarupa y Goyal, 2020). El emprendimiento se ha convertido así en un fenómeno de vital importancia para nuestras sociedades, lo que explica el gran interés que ha suscitado (Sánchez-García et al., 2017). Ahora bien, en el último tiempo se le ha venido brindando cada vez mayor atención a un tipo particular de emprendimiento, distinto del emprendimiento comercial o tradicional: se trata del denominado "emprendimiento social" (Mair y Martí, 2006; Sassmannshausen y Volkmann, 2018; Singh, 2016; Short, Moss y Lumpkin, 2009).

El creciente interés por esta variante del emprendimiento se explica en gran medida por las múltiples crisis que han aquejado a diversas naciones alrededor del mundo, lo que ha llevado a depositar grandes esperanzas en este tipo de iniciativas para la solución de los acuciantes problemas sociales que éstas han traído consigo (Enciso, Gómez y Mugarra, 2012; Kostetska y Berezyak, 2014; Vernis y Navarro, 2011). Es así como, desde diferentes sectores, las empresas con fines sociales -que aquí identificamos con las empresas pertenecientes a la Economía Social- han sido consideradas como una respuesta innovadora, dinámica, inclusiva y autosostenible a los principales desafíos sociales, económicos y medioambientales que enfrentan las sociedades actuales, muchos de los cuales han sido desatendidos -ya sea por incapacidad y/o desinteréstanto por los gobiernos y las instituciones públicas como por el sector privado (Caballero, Fuchs y Prialé, 2014; Comisión Europea, 2016; Defourny, Hulgard y Pestoff, 2014; Farber, Caballero, Prialé y Fuchs, 2015; Santos, 2012).

Es justamente en virtud del relevante impacto social y económico que generan las iniciativas empresariales en el ámbito del emprendimiento social, que apoyar a los emprendedores sociales se ha convertido en una necesidad (Defourny et al., 2014; Vernis y Navarro, 2011). Lo anterior se ha visto reflejado en la puesta en marcha de una serie de políticas públicas destinadas a favorecer las actividades emprendedoras de carácter social (Comisión Europea, 2016; Gómez, Mira y Martínez, 2007). Como apuntan Vernis y Navarro (2011), de la promoción del éxito y la expansión de las ideas de los emprendedores sociales dependen en gran medida el nivel de competitividad de los países y el mejoramiento de las condiciones productivas y de vida de sus ciudadanos.

Ahora bien, a pesar del gran interés que ha despertado el emprendimiento social en círculos académicos, profesionales y políticos, esta noción sigue siendo problemática; si bien la literatura sobre este fenómeno ha experimentado un notable desarrollo en los últimos años, aun no existe una definición unificada en torno a esta realidad económica (Cukier, Trenholm, Carl y Gekas, 2011; Lee, Battilana y Wang, 2014; Mair y Martí, 2006; Short et al., 2009). Algunos de estos aspectos nebulosos se relacionan, entre otras cuestiones, con cómo se diferencia el emprendimiento social del emprendimiento comercial o clásico, y con cuál es el rol que desempeñan los individuos en el proceso emprendedor social y en la creación de empresas con fines sociales.

En cuanto al primero de estos puntos, en la literatura sobre emprendimiento se ha venido dando un amplio debate entre quienes señalan que el emprendimiento comercial no debería distinguirse del emprendimiento social -pues a juicio de algunos autores ambos estarían generando valor social (Acs, Bosma y Sternberg, 2011)-, y aquellos que plantean que este último presenta ciertas características particulares que lo diferencian claramente del emprendimiento convencional (Austin, Stevenson y Wei-Skillern, 2006). Si bien existe un relativo consenso en cuanto a que los dos tipos de emprendimiento generan valor social y presentan otras características comunes, lo cierto es que la mayor parte de la literatura reconoce que uno y otro son diferenciables entre sí, fundamentalmente desde el punto de vista de la misión última de la empresa creada y de las características de la actividad emprendedora (Dees, 1998; Guzmán y Trujillo, 2008; Lepoutre, Justo, Tergensen y Bosma, 2011). Aquí se plantea que, más de allá de sus similitudes, se puede hablar de diferencias importantes entre uno y otro tipo de emprendimiento, y que dichas diferencias se plasmarían en la configuración distintiva de las características de quien está detrás de un emprendimiento de tipo social: la figura del emprendedor social. No es posible hablar de emprendimiento social sin referirse al emprendedor social, quien es en definitiva el que traduce una idea o una oportunidad en una empresa concreta (Enciso et al., 2012).

En general, en la literatura se reconocen en los emprendedores sociales una serie de atributos y virtudes, como el ser dinámicos, creativos y eficientes (Dees, 1998; Nissan, Castaño y Carrasco, 2012). Además, se destaca en ellos la posesión de fuertes valores sociales, lo que les permite constituirse como agentes de cambio, como líderes creíbles e íntegros, capaces de aunar voluntades tras su proyecto (Austin et al., 2006). 
Los emprendedores sociales se plantearían como propósito reformar la sociedad, no conformándose con solucionar problemas sociales de manera puntual; al contrario, lo que desean es producir cambios sostenibles en el tiempo, a la vez que replicables (Santos, 2012). En este sentido, en las acciones de los emprendedores sociales existe un potencial de cambio en las vidas de un gran número de personas (Alvord, Brown y Letts, 2004; Seelos y Mair, 2005).

La figura del emprendedor social resulta entonces tremendamente relevante por cuanto, frente a las diversas crisis económicas, sociales y ecológicas que sufren muchos territorios y países en el mundo, se hace cada vez más necesario que surjan individuos capaces de enfrentar estos problemas de manera creativa e innovadora. Por otra parte, si se conoce con mayor exactitud su perfil, dicha información podría ser utilizada ya sea para crear o mejorar programas ya existentes de formación de emprendedores (Marulanda et al., 2014), la mayoría de los cuales no contemplan la educación específica en emprendimiento social (Atxabal, 2014; Bel Durán, Fernández, García, Lejarriaga y Martin, 2016). Esto es de particular importancia en el caso de la población más joven, pues es precisamente la juventud la fuente más prometedora de iniciativas emprendedoras con que un país puede contar (Unger, Rauch, Frese y Rosenbusch, 2011; Uriarte y Martin, 2007).

El presente trabajo pretende ser un aporte en este sentido al tener como propósito principal caracterizar y comparar a los estudiantes que participan de un proceso formativo en emprendimiento dirigido a jóvenes entre los 16 y 24 años impartido en los Centros de Formación Profesional de la Comunidad Autónoma del País Vasco (CAPV), España. Concretamente, se identifican sus principales atributos personales, los cuales podrían contribuir a configurar intenciones de emprender en estos jóvenes, sean ellas en el ámbito del emprendimiento comercial o social. Asimismo, se busca determinar cuáles son los atributos personales que se relacionan en mayor medida con intenciones de emprender socialmente, vale decir, con la creación de empresas de la Economía Social. Se plantea aquí como hipótesis que la principal diferencia entre potenciales emprendedores sociales y comerciales se da en relación con la posesión de ciertos valores sociales, en especial la solidaridad y el sentido ético, los cuales se asociarían en mayor medida con el emprendimiento social y la Economía Social que con el emprendimiento convencional.

Han sido numerosos los modelos explicativos que, desde distintas perspectivas, han pretendido dar cuenta de la conducta emprendedora (Alonso y Gálvez, 2008; Pedrosa, 2015). En particular, en este estudio nos centramos en la "teoría de la personalidad", con el objeto de evaluar si esta aproximación presenta valor explicativo respecto de la población escolar, la cual ha sido prácticamente inexplorada, pues la mayor parte de las investigaciones sobre atributos de personalidad emprendedores se han realizado con muestras de estudiantes universitarios (Palamida, 2016; Valencia y Marulanda, 2019). Asimismo, una de las características centrales de la investigación realizada hasta la fecha en el ámbito del emprendimiento social ha sido su marcado énfasis en el aspecto conceptual, existiendo pocos estudios de carácter empírico y con muestras amplias (Miller y Wesley; Short et al., 2009). Es así como este estudio representa un esfuerzo inédito, por cuanto se trata de una investigación empírica de gran alcance en relación con una población poco estudiada.

El curso de formación del que participa la población objetivo de este estudio se denomina Ikasenpresa, y consiste en un programa educativo coordinado por TKNIKA o Centro de investigación e Innovación Aplicada para la Formación Profesional de la CAPV. El programa se imparte en el módulo de empresa e iniciativa emprendedora, y una de sus actividades centrales consiste en que los estudiantes deben crear una empresa que es utilizada como herramienta de aprendizaje, lo que permite diferenciar a los jóvenes según sus iniciativas correspondan al área del emprendimiento comercial o social. Ikasenpresa va en línea con el IV Plan Vasco de Formación Profesional, uno de cuyos objetivos es reforzar la actividad emprendedora, ya que en este programa participan la mayoría de los centros públicos y concertados de Euskadi ${ }^{3}$.

\section{La Teoría de la Personalidad como explicación del comportamiento emprendedor en los jóvenes}

Se han desarrollado varios modelos teóricos enfocados en las características de personalidad en tanto determinantes de la intención o conducta emprendedora (Brandstatter, 2011). Por su parte, el poseer intenciones de emprender ha sido ampliamente aceptado como un prerrequisito fundamental de la actividad emprendedora, sea ésta social o comercial (Kolvereid y Isaksen, 2006; Mair y Noboa 2006; Nurdan y Nancy, 2016).

Las distintas aproximaciones que vinculan rasgos de personalidad e intenciones de emprender caben dentro de lo que se ha denominado como "teoría de la personalidad" o "teoría de los rasgos", perspectiva

Específicamente, sus objetivos principales son: i) La creación de una Ikasenpresa, con lo que el alumnado toma consciencia de lo que implica emprender y los pasos que tienen que dar para ello; ii) Desarrollar las competencias de los jóvenes para ayudar a convertirlos en buenos profesionales y personas activas; y iii) Sensibilizarlos para que consideren el autoempleo como una opción más (Ministerio de Educación, Cultura y Deporte, 2015) 
teórica que se ha constituido como una de las líneas de investigación mejor establecidas dentro del campo de estudio de los emprendedores (Rauch y Frese, 2007b). La teoría de la personalidad se basa en tres supuestos principales, a saber: i) El emprendimiento juega un rol central en la generación de resultados relevantes o deseables, tales como la innovación, el crecimiento económico y la creación de empleo (Kirzner, 1997; Reynolds, Bygrave y Autio, 2004); ii) Los emprendedores, a diferencia de otros individuos o al menos en mayor medida que el resto de la población, desarrollan ciertas conductas o llevan a cabo ciertas actividades o acciones que contribuyen a estos resultados deseables (Baron y Henry, 2010); iii) Estos comportamientos y actividades están determinados -al menos parcialmente- por algunas características de personalidad o habilidades personales (Rauch y Frese, 2007b; Sánchez-García et al., 2017).

Ewen (2010) señala que la personalidad se origina dentro del individuo y describe las características centrales -y relativamente estables- que dan cuenta de patrones de comportamiento consistentes. En este sentido, la personalidad incluye aspectos mentales, emocionales, sociales y físicos que son observables/no observables y conscientes/inconscientes. Los rasgos se consideran unidades fundamentales y estables, integradas en la personalidad del individuo, los cuales determinan los comportamientos individuales en una amplia variedad de situaciones (Smith, 1999). De este modo, entonces, los rasgos varían entre los individuos influyendo, consecuentemente, en sus respectivas conductas. En el ámbito específico del emprendimiento, el emprendedor ha sido determinado y diferenciado del resto de la población por un conjunto de características de personalidad y comportamientos relacionados con actividades y cursos de acción emprendedores (Gartner, 1988; Rauch y Frese, 2007b; Sánchez-García et al., 2017). Las investigaciones se han centrado en gran medida en el papel de las características personales que configuran la personalidad de los emprendedores o la "personalidad emprendedora", y que por ende pueden predecir el comportamiento emprendedor (Rauch y Frese, 2007a).

Una línea de investigación se ha centrado en características amplias, como son los rasgos de personalidad conocidos como los Big-Five, los cuales señalan el rol de la responsabilidad, la apertura, la estabilidad emocional, la extroversión y la amabilidad en la predicción de la conducta emprendedora (Brandstatter, 2011; Wang, Chang, Yao y Liang, 2016). Junto con este tipo de trabajos centrados en factores generales o agregados, existe una línea de investigación más frecuente, la cual propone múltiples rasgos específicos como propios de la personalidad emprendedora (Rauch y Frese, 2007b). Este tipo de estudios surgen en parte como respuesta a las limitaciones que presentan los modelos basados en los Big-Five, en la medida en que estos constituyen constructos excesivamente amplios, los cuales podrían predecir un conjunto general de comportamientos, pero no así conductas específicas como las que llevan a iniciar una empresa (Baum y Locke, 2004; Rauch y Frese, 2007b).

Se han llevado a cabo diversos estudios meta-analíticos que han brindado evidencia respecto de la validez predictiva de diferentes aspectos de la personalidad a la hora de analizar la conducta emprendedora (Brandstatter, 2011; Rauch y Frese, 2007b; Zhao y Seibert, 2006; Zhao, Seibert y Lumpkin, 2010). Del lado de los Big-Five, diferentes autores han señalado la existencia de un vínculo entre estos cinco rasgos de la personalidad y la conducta emprendedora, en particular de la responsabilidad y de la apertura a la experiencia, características que presentan una mayor relación tanto con el comportamiento emprendedor como con el rendimiento empresarial (Farrington, 2012; Yim y Weston, 2007; Zhao et al., 2010; Zhao y Seibert, 2006). Ahora bien, Zhao et al. (2010), en su revisión meta-analítica basada en 60 estudios relacionados con los rasgos de personalidad de los Big-Five, apuntan que la magnitud de la relación es moderada. El campo de los rasgos de personalidad específicos, por otro lado, ha sido más prolífico en comparación al de los Big-Five, presentando esta perspectiva un mayor poder explicativo (Pedrosa, 2015). Las investigaciones se han centrado abrumadoramente en muestras de estudiantes universitarios (Palamida, 2016; Valencia y Marulanda, 2019) en desmedro de otro tipo de poblaciones -por ejemplo, muestras de estudiantes escolares-, concluyendo que aquellos estudiantes que presentan altos niveles en rasgos tales como autoeficacia, necesidad de logro, propensión al riesgo, locus de control interno, tolerancia a la ambigüedad, necesidad de innovación, proactividad, creatividad, optimismo y tolerancia al stress estarían más inclinados hacia el emprendimiento, esto al formar intenciones más fuertes respecto de la creación de nuevas empresas (Abir, Eyad y Zharan, 2014; Altinay, Madanoglu, Daniele y Lashley, 2012; Crespo, Belchior y Costa, 2018; Darmanto y Yuliari, 2018; Naushad y Malik, 2018; Newman, Obschonka, Schwartz, Cohen y Nielsen, 2018; Pihie y Bagheri, 2018; Prabhu y Poulose 2012; Prakash, Jain y Chauhan, 2015; Sesen, 2013; Tyszka, Cieslik, Domurat y Macko, 2011; Wang, Wang y Chen, 2017; Zinga, Coelho y Carvalho, 2013).

En el ámbito específico de grupos etarios de menor edad, por su parte, Frank, Lueguer y Korunka (2007) establecieron que los estudiantes secundarios con mayores niveles de necesidad de logro e innovación presentan intenciones emprendedoras más fuertes. Asimismo, Volery, Muller, Oser, Naepflin y Del Rey (2013) muestran que los estudiantes secundarios en Suecia que han participado en programas de formación emprendedora efectivamente forman intenciones de creación de nuevas empresas, esto independientemente de su necesidad de éxito y propensión a la innovación. La influencia de la propensión a asumir riesgos sobre las intenciones emprendedoras difiere en función de si los estudiantes están al principio o al final del curso 
sobre emprendimiento. Al comienzo del curso, la propensión al riesgo de los estudiantes no afecta las intenciones, lo que sí ocurre al final.

\section{El concepto de emprendimiento social y sus subconceptos}

Algo evidente cuando se habla de emprendimiento social es que no se puede hablar de él sin referirse a otros términos, al punto que muchas definiciones son inseparables, por ejemplo, de las definiciones de emprendedor social y de empresa social. Y es que el concepto de emprendimiento social tiene un carácter internamente complejo, el cual ha sido reconocido por varios autores, quienes lo describen como un concepto multidimensional y multifacético (Bacq y Janssen, 2011; Nicholls, 2008; Weerawardena y Sullivan-Mort, 2006). Así, para comprender el carácter internamente complejo del emprendimiento social, los investigadores han intentado identificar sus múltiples componentes. Se ha sugerido que el concepto de emprendimiento social consta de cinco componentes principales que contribuyen a su complejidad interna: creación de valor social, orientación de mercado, innovación social, organización de emprendimiento social y emprendedor social (Bacq y Janssen, 2011). Dado que los cinco componentes identificados son conceptos en sí mismos, se han entendido como "subconceptos" del emprendimiento social, cada uno de los cuales representa una parte integral de este fenómeno.

\subsection{Creación de valor social}

Un aspecto altamente valorado del emprendimiento social, el que se considera un requisito previo para que un emprendimiento sea calificado como tal, es la creación de valor social (Austin et al., 2006; Dees 1998; Peredo y McLean, 2006; Sharir y Lerner, 2006). Este aspecto se ha abordado más a fondo en las descripciones del emprendimiento social en tanto emprendimiento que tiene principalmente una misión social (Dees, 1998; Nicholls, 2008; Seelos y Mair, 2005; Sullivan-Mort, Weerawardena y Carnegie, 2003), que crea riqueza social (Zahra, Gedijlovic, Neubaum y Schulman, 2009), y que aborda problemas y necesidades sociales urgentes (Alvord et al., 2004; Bornstein, 2004; Light, 2006; Mair y Martí, 2006; Seelos y Mair, 2005). Por otra parte, el concepto de creación de valor social es un concepto cargado de valor e implica el "comportamiento virtuoso" (Sullivan-Mort et al., 2003), los objetivos altruistas (Tan, Williams y Tan, 2005) y la promoción de un propósito social que implica valores como la libertad, igualdad y tolerancia (Murphy y Coombes, 2009).

\subsection{Orientación de mercado}

El emprendimiento social tiene un doble objetivo: un objetivo social, es decir, busca solucionar problemas sociales, y un objetivo de sostenibilidad de la empresa (Austin et al., 2006; Curto, 2012; Dees, 1998; Haugh, 2005; Johnson, 2000; Mair y Marti, 2006; Martín y Osberg, 2007; Nicholls, 2006; Weerawardena y Sullivan, 2006; Zahra et al., 2009). El aspecto de la orientación de mercado del emprendimiento social se asocia así con la idea de una mayor eficiencia y eficacia a través de actividades comerciales (Nicholls, 2010) y la sostenibilidad financiera y la autosuficiencia de la organización (Harding, 2004; Haugh, 2005). Un aspecto clave que diferencia al emprendimiento social de la prestación tradicional de servicios sociales sin ánimo de lucro es justamente su foco implícito en la eficiencia y el uso efectivo de los recursos (Nicholls y Cho, 2006).

\subsection{Innovación social}

Nicholls y Cho (2006) afirman que es el enfoque no tradicional y disruptivo del emprendimiento social una de las características que también lo distingue de la prestación de servicios sociales tradicionales. Dees (1998), por su parte, afirma que los emprendedores sociales se involucran en un proceso de innovación continua, mientras que Peredo y McLean (2006) consideran que el emprendimiento social implica necesariamente el empleo de la innovación. En la misma línea, otros autores enfatizan que el emprendimiento social es una actividad innovadora de creación de valor social (Austin et al., 2006) que crea nuevos modelos (Seelos y Mair, 2005), y que un enfoque innovador para alcanzar la misión social es un elemento constituyente de este fenómeno (Nicholls, 2008). Muy relacionada con la idea de innovación está la idea de cambio; así, los emprendedores sociales han sido considerados como innovadores en la esfera social que impulsan cambios sociales importantes (Mair y Marti, 2006), provocan transformación social sostenible (Alvord et al., 2004) y ruptura de patrones (Martin y Osberg, 2007). 


\subsection{Organización del emprendimiento social}

Por lo general, las actividades que denominamos como emprendimiento social se organizan a lo largo del tiempo dentro de un marco organizativo. Según Mair y Marti (2006), es justamente este contexto organizativo en el que se produce el emprendimiento social el que distingue a este fenómeno de otras iniciativas dirigidas al cambio social, como son los movimientos activistas.

Tradicionalmente se ha identificado a la empresa social como la organización más representativa del emprendimiento social. A pesar de la falta de claridad conceptual existente respecto de lo que es la empresa social (Dart, 2004; Galera y Borzaga, 2009; Kruse, 2020), existen ciertos puntos de consenso en la literatura sobre el tema. A menudo se entiende que la noción de empresa social -en tanto organización que provee servicios y bienes de interés general y para la cual la maximización del beneficio ya no es una condición esencial- vino a romper con el concepto clásico de empresa, es decir, representa un cuestionamiento a la concepción compartida de las empresas como organizaciones que promueven el interés exclusivo de sus propietarios (Galera y Borzaga, 2009). En este sentido, la empresa social suele pensarse como algo "nuevo" y "distinto" respecto de los negocios clásicos y de la actividad no lucrativa tradicional, combinando en diferentes grados elementos del propósito social, la orientación de mercado y los estándares de rendimiento financiero de los negocios (Young, 2008). Por su parte, Peattie y Morley (2008) identificaron que, a pesar de la gran cantidad de definiciones existentes en torno a este concepto, se pueden rescatar dos características definitorias de todas ellas: la centralidad del comercio (comerciar para recibir ingresos) y la primacía de los objetivos sociales. Otro punto respecto del cual parece existir cierto consenso o aceptación es que la empresa social no necesariamente constituye una nueva forma organizacional, sino que más bien ha evolucionado desde formas tempranas de organizaciones no lucrativas, cooperativas y negocios tradicionales (Defourny y Nyssens, 2006).

Si bien existen muchas definiciones distintas de empresa social, es posible identificar dos tendencias o aproximaciones principales en torno a este fenómeno: la europea continental y la anglosojana o estadounidense (Galera y Borzaga, 2009; Kerlin, 2006). La primera de estas corrientes -conocida como enfoque EMES- ubica a las empresas sociales dentro del ámbito específico de la Economía Social. Si bien las empresas sociales presentan ciertas características que las diferencian de las organizaciones típicas de la Economía Social -cooperativas, asociaciones y mutualidades-, al mismo tiempo comparten con ellas ciertos principios fundamentales -por ejemplo, su autonomía y naturaleza democrática- que vinculan a unas y otras configurando así un todo distinto tanto de las empresas del sector público como de las del sector capitalista privado (Monzón y Chaves, 2012, 2017). En esta concepción, el concepto de Economía Social es mucho más amplio que el de empresa social, en la medida en que este último representa solo a una pequeña parte del primero; vale decir, la Economía Social comprende a un conjunto de agentes económicos, entre los cuales se cuentan, entre otros, las empresas sociales. Es así como, de acuerdo con esta visión, todas las empresas sociales son parte de este sector, pero la mayoría de las empresas de la Economía Social no forman parte del grupo de las empresas sociales (Monzón y Chaves, 2012).

\subsection{Emprendedor social}

Muchos autores han considerado al empresario o emprendedor social como el elemento central en el emprendimiento social (Bornstein, 2004; Dees, 1998; Leadbeater, 1997; Thompson, 2002). Del análisis de las definiciones de emprendedor social se desprende que se trata de una persona que decide crear una empresa con una misión social explícita en mente para el logro de una transformación social, siendo ésta la medida de su éxito (Dees, 1998; Nissan et al., 2012). Asimismo, para la creación de este valor social los emprendedores sociales desarrollan actividades empresariales de maneras innovadoras (Dees, Emerson y Economy, 2001; Santos, 2012; Zhara et al., 2009), casi siempre cubriendo nichos desatendidos por gobiernos e instituciones públicas (Santos, 2012; Thompson, Alvy y Lees, 2000).

Entre las definiciones más influyentes de emprendedor social se encuentra la de Dees (1998). Este autor afirma que los emprendedores sociales, al adoptar una misión social, crean valor social reconociendo y buscando sin descanso nuevas oportunidades, continuamente innovando, adaptándose y aprendiendo, y actuando audazmente sin verse limitados por los recursos disponibles. Los emprendedores sociales se caracterizarían además por exhibir una mayor responsabilidad hacia los beneficiarios de su acción accountability o rendición de cuentas- y los resultados generados. Para Dees (1998), los emprendedores sociales constituyen una clase especial de líderes. La definición provista por este autor incluye los elementos clave de la creación de valor social, la identificación y explotación de oportunidades por un fin social, y la innovación e inventiva/iniciativa para crear un cambio social. Este último aspecto resulta clave, y alude a la idea de que el emprendedor social no permite que su eventualmente limitada disposición de recursos iniciales constriña la puesta en marcha de una iniciativa empresarial (Peredo y McLean, 2006).

Posteriormente, otros autores agregaron nuevas dimensiones a la definición de emprendedor social de Dees (1998). Es el caso, por ejemplo, de la noción de tolerancia al riesgo (Brinckerhoff, 2000; Elkington y 
Hartigan, 2008; Tan, Williams y Tan, 2005; Weerawardena y Mort-Sullivan, 2006). Al respecto, Brinckerhoff (2000: 1) señala que "los emprendedores sociales son personas que toman riesgos en nombre de las personas a las que sirve su organización. Los emprendedores tradicionales toman riesgos en su propio nombre, o en nombre de los accionistas de su compañía. En las organizaciones no lucrativas, los riesgos son tomados en nombre de las partes interesadas". Así, los emprendedores sociales asumen riesgos por fines sociales o por una misión social. Más aún, para este autor el emprendimiento social se esfuerza por crear valor social, y en esta tarea, además de asumir riesgos, los emprendedores sociales deben desplegar innovación y proactividad.

La revisión de la literatura muestra que el conocimiento existente sobre el emprendedor social es mayoritariamente conceptual. Los estudios sobre emprendimiento social y emprendedores sociales se encuentran centrados mayoritariamente en aspectos relativos a la definición y, por consiguiente, en lo conceptual más que lo empírico (Dacin, Dacin y Tracey, 2010; Short et al., 2009). Con la excepción de unos pocos estudios cuantitativos, no se han llevado a cabo investigaciones con muestras amplias (Kruse, 2020; Miller y Wesley, 2010; Cukier et al., 2011). En cuanto a los rasgos de personalidad que se asociarían a los emprendedores sociales -muchos de los cuales también se vinculan con los emprendedores comerciales o convencionales- se han destacado, entre otros, la autoeficacia, el compromiso, la tolerancia al riesgo, la creatividad, el carisma y el liderazgo, la proactividad, la capacidad de generar confianza y credibilidad, la amabilidad y autoexigencia en el trabajo, la fortaleza ante las dificultades, la innovación y la sensibilidad a la exclusión, la marginación o al sufrimiento de aquellos que carecen de medios económicos o influencia política (Austin et al., 2006; Dees, 1998; Guzmán y Trujillo, 2008; Hockerts, 2015; Jiao, 2011; Mair y Noboa, 2006; Martín y Osberg, 2007; Nga y Shamuganathan, 2010; Sullivan-Mort et al., 2003; Thompson et al., 2000; Weerawardena y Sullivan-Mort, 2006; Zahra et al., 2009).

Si bien emprendedores sociales y emprendedores comerciales comparten características comunes, en los primeros se ha destacado su vínculo con fuertes valores sociales tales como la solidaridad y un especial sentido ético, esto en virtud del tipo de fines que persiguen y por su deseo de provocar cambios significativos que sean perdurables y sustentables en el tiempo (Curto, 2012). Es así como se esperaría que las principales diferencias en cuanto a atributos entre emprendedores sociales y comerciales se diera en relación con estas variables.

\section{Metodología}

La presente investigación es de tipo exploratoria-descriptiva, por cuanto se propone describir y extraer perfiles de una población poco explorada en relación con una serie de variables referidas fundamentalmente a atributos emprendedores, los cuales se vincularían con la configuración de intenciones de emprender, ya sea a través de la creación de una empresa comercial o social.

La población objetivo de este estudio está conformada por los estudiantes de formación profesional de grado medio y superior de la CAPV que cursan el módulo de "Empresa e iniciativa emprendedora" (matriculados en el año lectivo 2017-2018) en el segundo curso (segundo año de estudios) de centros públicos y privados adscritos al programa educativo Ikasenpresa. El tipo de muestreo fue de carácter censal, vale decir, se intentó encuestar a la totalidad de matriculados en el módulo anteriormente mencionado. De un total de 6.571 matriculados para el período 2017-2018, se logró encuestar a 5.767 estudiantes, es decir, al $87,76 \%$.

Fueron aplicadas dos encuestas de elaboración propia a la muestra de estudiantes: un cuestionario principal o largo que incluyó preguntas de caracterización sociodemográfica, así como una escala tipo Likert de autopercepción de atributos emprendedores; y una encuesta reducida o corta contestada solo por un subgrupo de la muestra total (participantes de la feria de emprendimiento). Para efectos del análisis, se definieron los siguientes grupos relevantes en términos comparativos:

i) Todos los respondientes de la encuesta principal, vale decir, la muestra total;

ii) Quienes respondieron la encuesta principal, pero que sin embargo no participaron de la feria de emprendimiento organizada por Ikasenpresa, vale decir, no presentaron una propuesta de empresa. A este grupo se le denomina como los "no emprendedores";

iii) Quienes respondieron la encuesta principal y al mismo tiempo participaron de la feria de emprendimiento, es decir, sí presentaron una propuesta de empresa. Este grupo corresponde a los "emprendedores potenciales", a los que a su vez se les aplicó el cuestionario corto destinado a indagar en las motivaciones de su proyecto empresarial y en el tipo y características de la empresa creada;

iv) Quienes respondieron la encuesta principal, participaron de la feria de emprendimiento y presentaron proyectos empresariales en el ámbito del emprendimiento comercial. Este es el grupo de los "emprendedores potenciales comerciales"; 
v) Quienes respondieron la encuesta principal, participaron de la feria de emprendimiento y presentaron proyectos empresariales en el área del emprendimiento social, pudiendo estos corresponder a cualquier organización dentro del ámbito de la Economía Social, incluidas las empresas sociales. Este es el grupo de los "emprendedores potenciales sociales".

El supuesto que subyace a este estudio es que la formación de intenciones de emprender es el resultado de los atributos personales, que incluyen tanto rasgos de personalidad como habilidades y competencias. Se entiende que quienes en mayor medida presentan intenciones de emprender futuras son los emprendedores potenciales, vale decir, los jóvenes que han presentado proyectos empresariales -comerciales o socialesdentro del marco de la feria de emprendimiento del programa Ikasenpresa de TKNIKA. El modelo propuesto puede sintetizarse en la siguiente Figura.

Figura. 1. Modelo propuesto

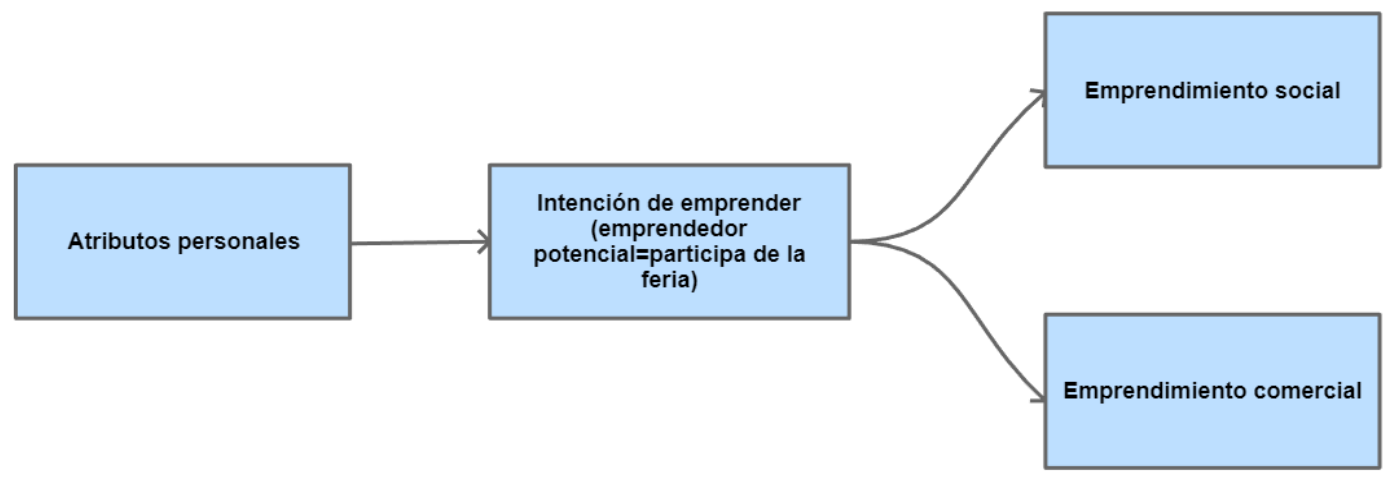

Fuente: Elaboración propia.

El análisis de los datos se llevó a cabo por medio de análisis descriptivo de frecuencias y porcentajes, comparaciones de medias y regresión logística, esta última técnica utilizada para establecer un modelo que permita establecer cuáles son los variables que aumentan la probabilidad de emprender socialmente.

\section{Resultados}

\subsection{Antecedentes generales de la muestra}

En esta investigación se incluyeron un total de 74 centros educativos del País Vasco, pertenecientes en su mayoría a la provincia de Bizkaia (44\%), seguida de cerca por Gipuzkoa (42\%) y, finalmente, Álava (15\%). De los 5.767 estudiantes encuestados, el 65\% corresponden a hombres y el 34\% a mujeres. El 39\% de los entrevistados se encontraba cursando grado medio al momento de la aplicación de la encuesta, en tanto que el $59 \%$ el grado superior.

Los estudiantes provienen fundamentalmente de familias en que la ocupación de la persona que aporta el mayor ingreso al hogar es de asalariado fijo (58\%). Solo el $2 \%$ es miembro de una cooperativa y el $8 \%$ se encuentra desempleado. Por su parte, los estudiantes en su mayoría han realizado labores para obtener recursos económicos propios, principalmente actividades eventuales (35\%) y/o actividades con contrato (36\%). Asimismo, la mayor parte de los estudiantes tiene experiencia o conoce de cerca empresas: el 58\% ha tenido la posibilidad de conocer más de dos empresas, el $64 \%$ ha trabajado o realizado prácticas laborales en empresas, y el $51 \%$ tiene algún familiar directo que posee negocio propio.

En cuanto a la intención de iniciar un negocio, poco más de la mitad de los encuestados (51\%) manifiesta que no ha pensado en la posibilidad de emprender en el futuro. Solo al $29 \%$ de los estudiantes les parece atractivo emprender por su cuenta con una empresa propia, mientras que el porcentaje restante $(71 \%)$ preferiría trabajar como subordinado o bien desarrollar una carrera relacionada con la investigación, el arte o el deporte. No obstante lo anterior, al $66 \%$ le parece motivante poseer un negocio propio. 


\subsection{Atributos personales en la muestra total}

Los atributos considerados en este estudio corresponden a características personales -rasgos de personalidad, habilidades, competencias- de los seres humanos que, en el ámbito del emprendimiento, han sido identificadas como factores clave a la hora de entender este fenómeno. El instrumento utilizado para medir atributos consistió en una escala de tipo Likert de cinco puntos -con las opciones de respuesta nada capaz (1); poco capaz (2); más o menos capaz (3); capaz (4); y muy capaz (5)- compuesta, a su vez, de 20 subescalas que miden diferentes características individuales. Tras una exhaustiva revisión bibliográfica, se relevaron los siguientes atributos como propios de los emprendedores comerciales y sociales, a saber: Autoconfianza (A1); Locus de control interno ${ }^{4}$ (A2); Propensión al riesgo (A3); Capacidad innovadora (A4); Tolerancia al estrés (A5); Optimismo (A6); Autonomía (A7); Motivación de logro (A8); Perseverancia (A9); Capacidad para identificar oportunidades (A10); Tolerancia a la ambigüedad (A11); Capacidad de comunicación (A12); Proactividad (A13); Capacidad para gestionar de recursos (A14); Capacidad de trabajo en equipo (A15); Capacidad de aprendizaje (A16); Capacidad de gestión de TICS (A17); Liderazgo (A18); Solidaridad (A19); y Ética (A20).

En un primer momento, se establecieron evidencias de validez y confiabilidad de la escala de autopercepción de atributos mediante análisis factoriales confirmatorios para variables categóricas, con correlaciones policóricas y estimadores robustos con cada uno de los atributos en estudio; posteriormente, se calculó la confiabilidad ordinal. Los resultados indican que las escalas reducidas tienen adecuado ajuste, así como adecuada evidencia de validez y de confiablidad. De un total de 102 ítems, fueron eliminados 16, quedando 86. Como es posible identificar en el Gráfico que se muestra a continuación, la mayor parte los estudiantes presentan en promedio más de 3 puntos -sobre un total de 5- en cada uno de los atributos relacionados con el emprendimiento. Cabe destacar que existe un promedio superior a los 4 puntos en los atributos locus de control interno (A2) y capacidad de aprendizaje (A16).

Gráfico. 1. Puntaje promedio de atributos (muestra total)

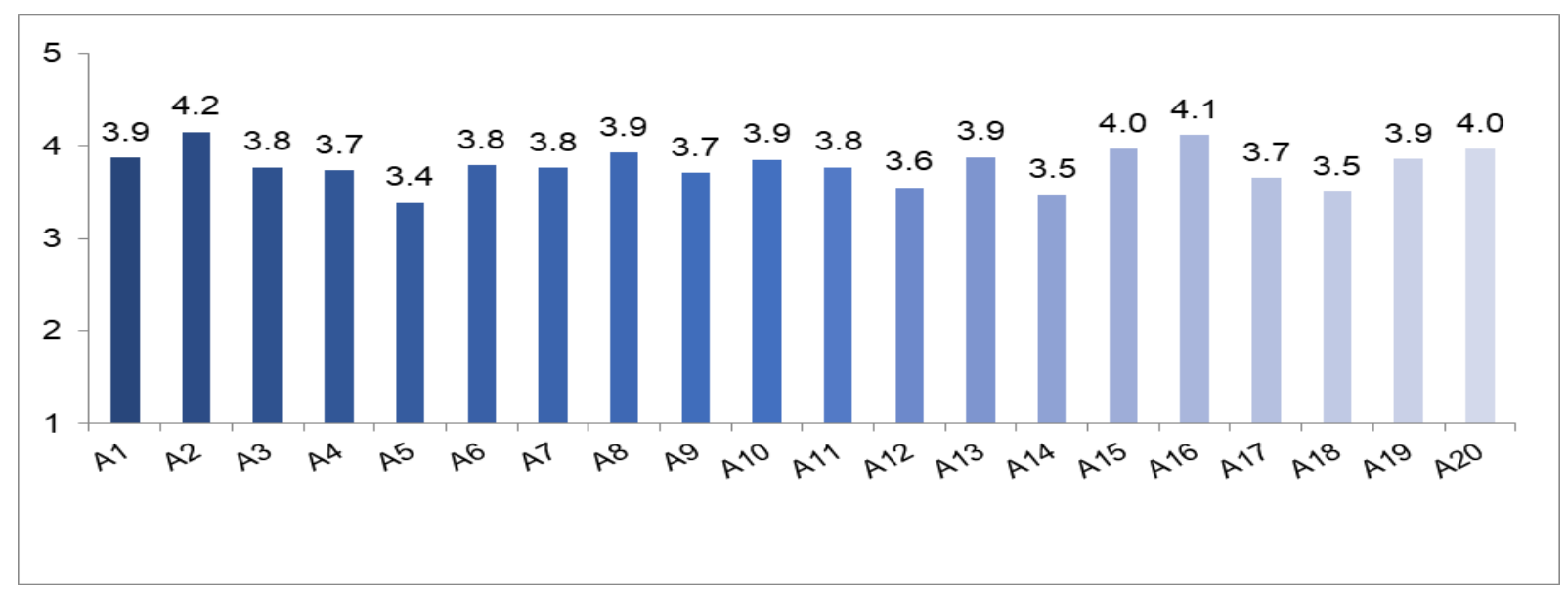

Fuente: Elaboración propia.

Cuando se comparan los atributos según sexo, edad y el grado que estaban cursando los estudiantes al momento de responder la encuesta, es posible identificar diferencias significativas entre hombres y mujeres en la mayoría de las variables, a excepción de la disposición a correr riesgos y la tolerancia al estrés. En este sentido, las mujeres tienden a presentar un promedio más alto que los hombres en la mayoría de las características individuales relevantes para el emprendimiento. Por otra parte, la edad produjo diferencias significativas en todas las variables, observándose una tendencia al aumento en los valores de los atributos a medida que ésta se acrecienta. Asimismo, quienes cursan el grado medio tienden a presentar menores puntajes promedio en los atributos testeados.

\subsection{Perfil general de no emprendedores y emprendedores potenciales}

Del total de la muestra de 5.697 estudiantes, fueron 377 (6\%) los estudiantes que participaron de la feria de emprendimiento organizada por Ikasenpresa. Este grupo lo hemos denominado aquí como "emprendedores potenciales". El 58\% de este conjunto corresponde a mujeres, mientras que el $42 \%$ a hombres. En cuanto al

4 El locus de control interno se refiere al grado o nivel en el cual un individuo percibe que los éxitos o los fracasos dependen de sus iniciativas personales (Palamida, 2016). 
grado cursado, el $37 \%$ estaba cursando grado medio, en tanto que el $62 \%$ el grado superior. En el caso de los no emprendedores o aquellos estudiantes que no participaron de la feria, el $67 \%$ son hombres y el $32 \%$ mujeres, mientras que el 59\% cursaba el grado superior y el 39\% el grado medio.

La mayor parte de los potenciales emprendedores indica que quien aporta con mayores ingresos en su hogar es asalariado fijo (57\%). Únicamente el $2 \%$ es miembro de una cooperativa y en el $7 \%$ de los casos se registra una situación de desempleo. Estos porcentajes son muy similares para el grupo de los no emprendedores. Por su parte, tanto no emprendedores como emprendedores potenciales en su gran mayoría sobre el 70\%- cuentan con experiencia laboral, especialmente en actividades eventuales. Uno y otro grupo también tienen experiencia o conocen de cerca empresas. En este sentido, el $65 \%$ de los emprendedores potenciales y el $63 \%$ de los no emprendedores han trabajado o realizado prácticas en empresas; el 57\% de los emprendedores potenciales y el 58\% de los no emprendedores han tenido la posibilidad de conocer más de dos empresas; y el 54\% de los emprendedores potenciales y el 51\% de los no emprendedores tienen algún familiar directo que posee negocio propio.

En cuanto a la intención y motivación por emprender, se registran ligeras diferencias a favor de los emprendedores potenciales. Por ejemplo, el $70 \%$ de estos versus el $66 \%$ de los no emprendedores considera motivante tener un negocio propio. Asimismo, el 52\% de los emprendedores potenciales ha pensado en llevar a cabo algún negocio en el futuro, mientras que este porcentaje en el caso de los no emprendedores es de un $47 \%$.

\subsection{Caracterización de las empresas de los emprendedores potenciales}

Los emprendedores potenciales debieron crear su propia empresa dentro del marco de la feria de emprendimiento de Ikasenpresa. El 83\% de estas empresas fueron pensadas como sociales -incluyendo cooperativas, asociaciones y fundaciones- y el $17 \%$ comerciales. Por su parte, las principales motivaciones para participar del concurso fueron lograr un objetivo colectivo (85\%), enfrentar un nuevo reto (85\%), vincular el emprendimiento con una actividad que los apasiona (85\%) y contribuir a la sociedad (83\%).

Entre las características de las empresas creadas destaca que en el $83 \%$ de los casos fueron pensadas para que cada integrante o socio tenga derecho a voto (una persona=un voto); en el 53\% la empresa crea nuevos bienes o presta servicios no ofrecidos por otros proveedores; y en el 51\% los bienes o servicios ofrecidos por la empresa son de carácter social, comunitario o ambiental.

En lo que refiere a la proyección a futuro del emprendimiento, los emprendedores potenciales buscan fundamentalmente que la empresa distribuya un porcentaje de sus ganancias entre todos los miembros del grupo (72\%) y que la mayor parte de los ingresos sea producto de la actividad comercial (71\%). Un porcentaje algo menor distribuiría las ganancias equitativamente (64\%), así como fomentaría la participación de todos los miembros de la organización (trabajadores, socios, clientes) (62\%) y reinvertiría las ganancias para mejorar la empresa $(57 \%)$.

\subsection{Atributos de no emprendedores y emprendedores potenciales}

Como se puede apreciar en el Gráfico 2, las únicas diferencias estadísticamente significativas encontradas en relación a los atributos entre el grupo de los no emprendedores y los emprendedores potenciales se observan en la capacidad de innovar (A4), la perseverancia (A9), la capacidad de identificar oportunidades (A10), la capacidad de trabajar en equipo (A15), la capacidad de aprendizaje (A16), la solidaridad (A19) y la ética (A20). En estas variables, los emprendedores potenciales tienen puntajes promedio superiores a los de los no emprendedores. Así también, se encontraron diferencias marginalmente significativas $(<0.1)$ en el locus de control interno (A2) entre quienes emprenden y quienes no emprenden, estos últimos con medias más bajas. 
Gráfico. 2. Emprendedores y no emprendedores según atributos personales

\section{Ética $(\mathrm{A} 20)^{*}$ \\ Solidaridad (A19)** \\ Liderazgo (A18) \\ Gestión de TICS (A17)}

Capac. de aprendizaje (A16)*

Trabajo en equipo (A15)*

Gestión de recursos (A14)

Proactividad (A13)

Comunicación (A12)

Tolerancia a la ambigüedad (A11)

Identificación de oportunidades (A10)*

$$
\begin{array}{r}
\text { Perseverancia (A9)*** } \\
\text { Motivación de logro (A8) }
\end{array}
$$

Autonomía (A7)

Optimismo (A6)

Tolerancia al estrés (A5)

Innovación (A4)*

Propensión al riesgo (A3)

Locus de control interno (A2)

Autoconfianza (A1) (.)
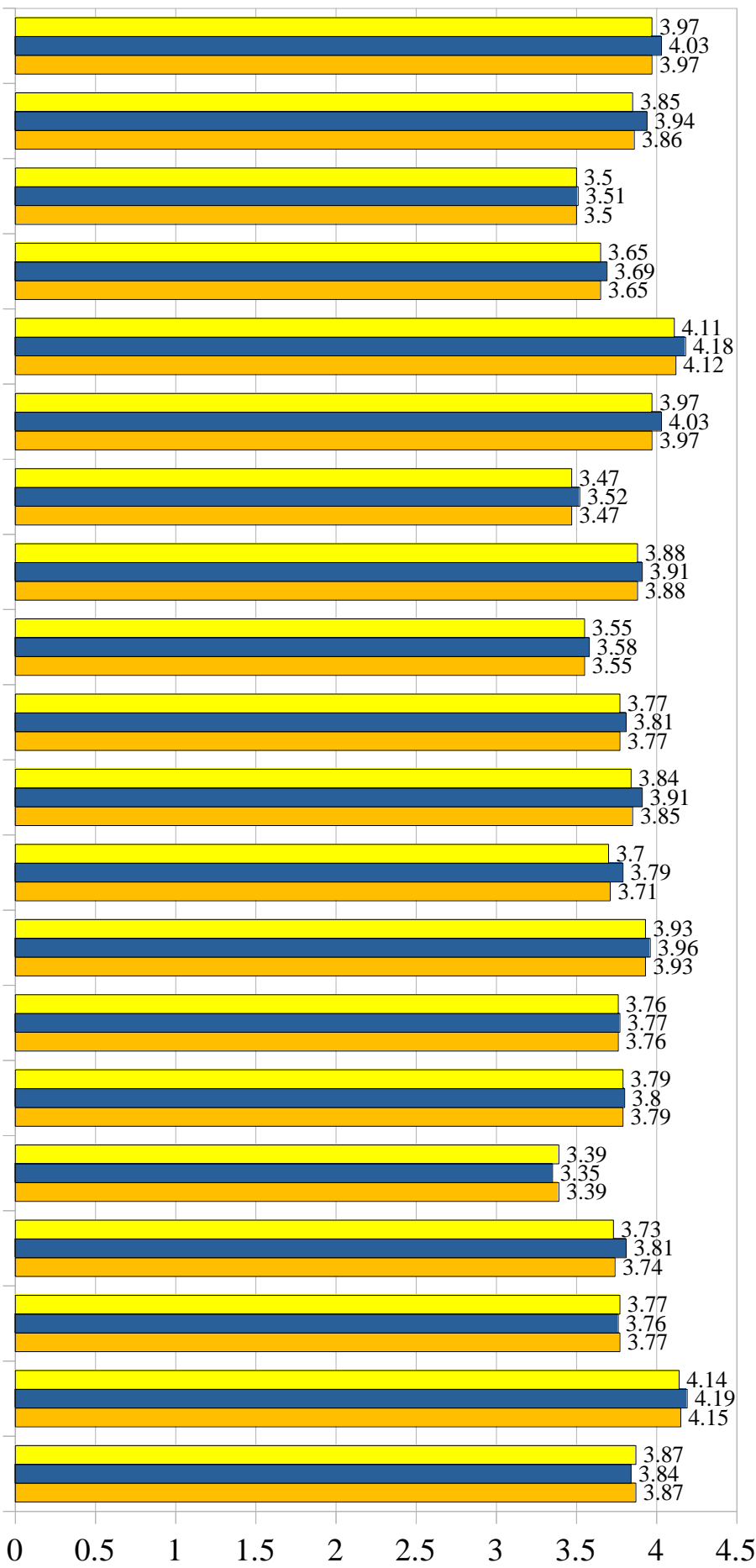

$\square$ No emprendedor $\square$ Emprendedor $\square$ Total

$$
\mathrm{p}<0.001 ; * * \mathrm{p}<0.01 ; * \mathrm{p}<0.05 ; \text { (.) } \mathrm{p}<0.1
$$

Fuente: Elaboración propia.

\subsection{Atributos de emprendedores potenciales comerciales y sociales}

Como se puede ver en el siguiente Gráfico, existen diferencias estadísticamente significativas entre emprendedores potenciales comerciales y sociales -en favor de los segundos- en prácticamente todos los atributos considerados en esta investigación. El único atributo donde no se registran diferencias significativas entre ambos grupos es en autoconfianza (A1). 
Gráfico. 3. Emprendedores potenciales comerciales y sociales según atributos personales

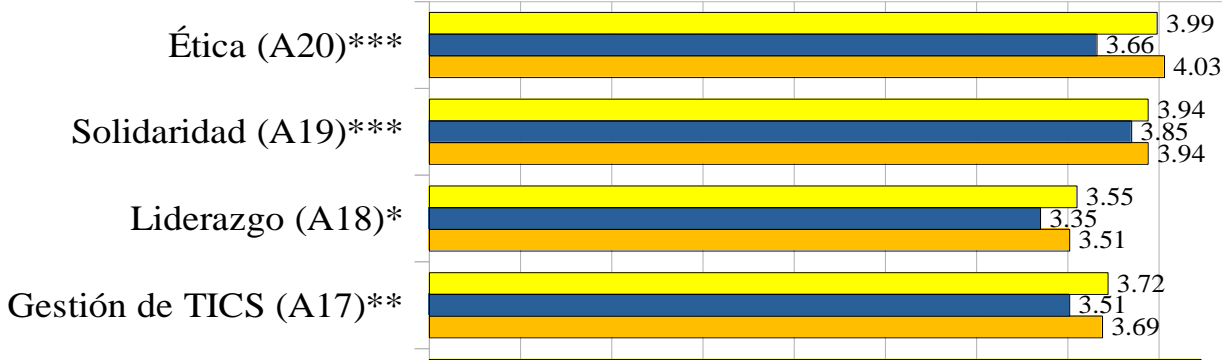

Capac. de aprendizaje (A16)***

Trabajo en equipo (A15)***

Gestión de recursos (A14)*

Proactividad $(\mathrm{A} 13) * *$

Comunicación (A12)*

Tolerancia a la ambigüedad (A11)***

Identificación de oportunidades (A10)***

Perseverancia (A9)***

Motivación de logro (A8)***

Autonomía (A7)***

Optimismo (A6)**

Tolerancia al estrés (A5)*

Innovación (A4)***

Propensión al riesgo (A3)*

Locus de control interno (A2)***
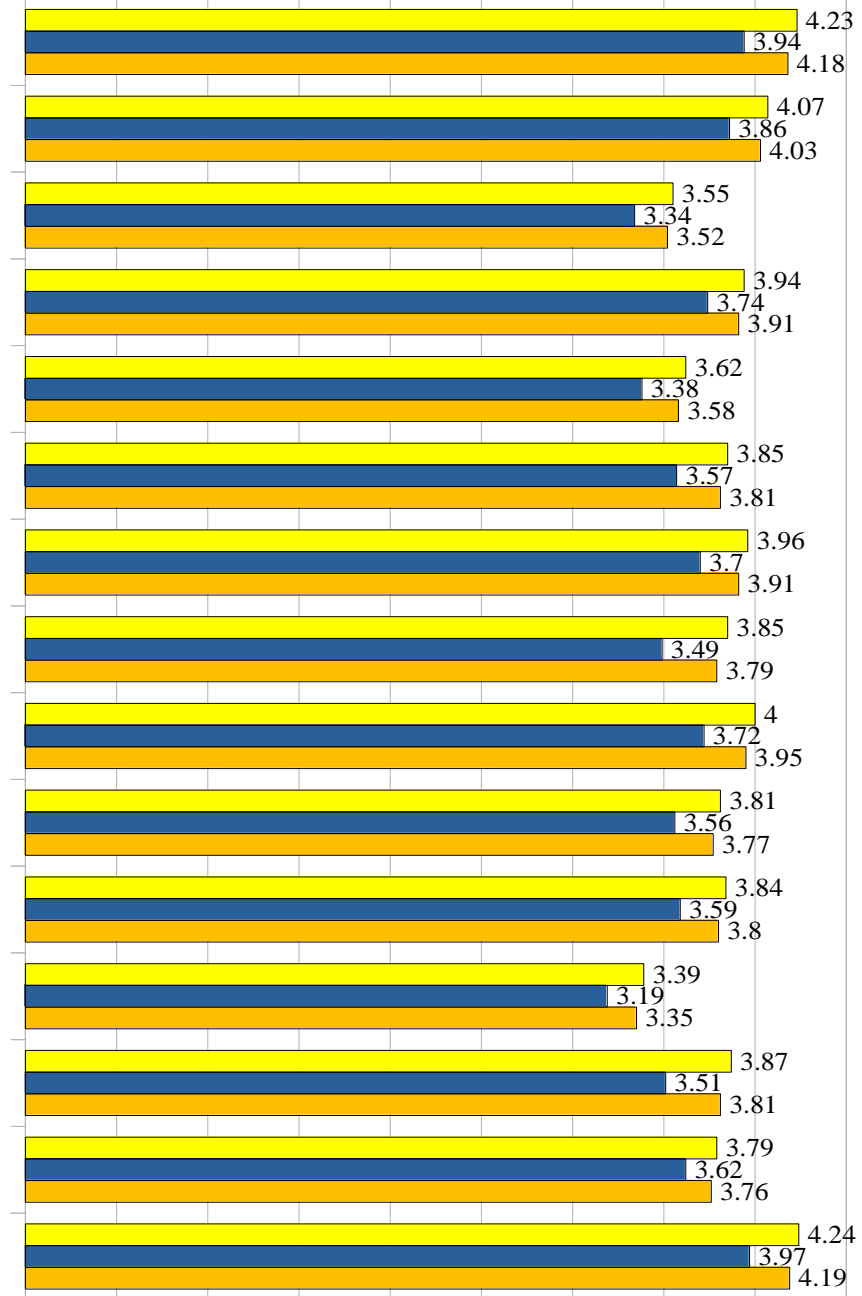

Autoconfianza (A1)

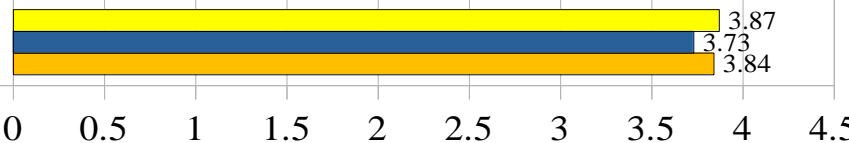

$\square$ Social $\square$ Comercial $\square$ Total

$$
\mathrm{p}<0.001 ; * * \mathrm{p}<0.01 ; * \mathrm{p}<0.05 ; \text { (.) } \mathrm{p}<0.1
$$

Fuente: Elaboración propia.

\subsection{Atributos que aumentan la probabilidad de emprender socialmente}

Con el fin de identificar los atributos que hacen más probable que el emprendimiento sea social, se llevó a cabo un análisis de regresión logística. Para ello, se incorporaron como variables independientes los atributos en los que se encontraron diferencias significativas entre emprendedores sociales y emprendedores comerciales, a saber: locus de control (A2), disposición a correr riesgos (A3) capacidad de innovar (A4), tolerancia al estrés (A5), optimismo (A6), autonomía (A7), motivación (A8), perseverancia (A9), capacidad de identificar oportunidades (A10), tolerancia a la antiguiedad (A11), habilidades comunicativas (A12), iniciativa/proactividad (A13), capacidad para gestionar recursos (A14), capacidad para trabajar en equipo 
(A15), capacidad de aprendizaje (A16), gestión de TICS (A17), liderazgo (A18), solidaridad (A19) y ética (A20). Estas variables fueron recodificadas en las categorías alto, medio y bajo. Para tal efecto, se sumaron los puntajes asociados a las respuestas a los ítems pertenecientes a cada atributo, para luego definir tres rangos de puntaje correspondientes a las categorías antes mencionadas. Para la construcción del modelo predictivo solo se incorporó la categoría "alto" de cada variable. Además, se incluyeron aquellas variables sociodemográficas en las que previamente se encontraron diferencias significativas entre los emprendedores sociales y comerciales: sexo, grado cursado y edad. Esta última variable fue recodificada en categorías y luego cada una de las categorías fueron transformadas en variables dummy (0/1) antes de ser incorporadas al modelo. Los resultados indican que sólo puntuar alto en solidaridad aumenta la probabilidad de emprender socialmente (Tabla 1).

Tabla. 1. Modelo de regresión logística

\begin{tabular}{|c|c|c|c|c|}
\hline & Logit & $\mathrm{Z}$ & $\mathrm{P}$ & \\
\hline Intercepto & 1.13 & 2.82 & 0.00 & $* *$ \\
\hline Mujer & 0.24 & 0.74 & 0.46 & \\
\hline Nivel Superior & -0.33 & -0.97 & 0.33 & \\
\hline$\leq 20$ años & 15.37 & 0.02 & 0.98 & \\
\hline 26-30 años & 0.23 & 0.68 & 0.50 & \\
\hline A2 Alto & 1.10 & 1.92 & 0.05 & \\
\hline A3 Alto & 0.50 & 1.05 & 0.29 & \\
\hline A4 Alto & 0.53 & 1.03 & 0.30 & \\
\hline A5 Alto & -0.51 & -1.16 & 0.25 & \\
\hline A6 Alto & -0.50 & -0.84 & 0.40 & \\
\hline A7 Alto & -0.10 & -0.21 & 0.84 & \\
\hline A8 Alto & 0.12 & 0.24 & 0.81 & \\
\hline A9 Alto & -0.18 & -0.35 & 0.72 & \\
\hline A10 Alto & -0.41 & -0.81 & 0.42 & \\
\hline A11 Alto & 0.10 & 0.20 & 0.84 & \\
\hline A12 Alto & -0.17 & -0.33 & 0.74 & \\
\hline A13 Alto & -0.14 & -0.25 & 0.80 & \\
\hline A14 Alto & 0.00 & 0.01 & 0.99 & \\
\hline A15 Alto & -0.14 & -0.30 & 0.76 & \\
\hline A16 Alto & 0.55 & 0.95 & 0.34 & \\
\hline A17 Alto & -0.29 & -0.59 & 0.56 & \\
\hline A18 Alto & -0.08 & -0.12 & 0.90 & \\
\hline A19 Alto & 1.83 & 2.68 & 0.01 & $* *$ \\
\hline A20 Alto & -0.02 & -0.04 & 0.97 & \\
\hline
\end{tabular}

El ajuste de este modelo adecuado ( $2 \mathrm{LL}=39,09 ; \mathrm{gl}=24 ; \mathrm{p}<0.05)$. La varianza explicada del modelo es el $10 \%$ de la muestra $\left(\mathrm{R}^{2}=0,179 ; \mathrm{R}^{2}\right.$ ajustado $\left.=0,099\right)$. Si analizamos las desviaciones es posible identificar que la desviación nula $(309,50 ; \mathrm{gl}=354)$ y residual $(270,41 ; \mathrm{gl}=330)$ tienen valores distintos, siendo esta diferencia no significativa ( $>$ >.05), lo que quiere decir que no podemos rechazar que los coeficientes sean cero. Dados estos resultados, se probó un modelo reducido, utilizando el método stepwise regression o regresión paso a paso, quedando aquellas variables que mejoran su ajuste (Tabla 2).

Tabla. 2. Modelo reducido

\begin{tabular}{ccccccccc}
\hline & Logit & Odds ratio & Prob & SE & $\mathrm{z}$ & $\operatorname{Pr}(>|\mathrm{z}|)$ & VIF \\
\hline (Intercepto) & 1.22 & 3.39 & 0.77 & 0.16 & 7.48 & 0.00 & $* * *$ & \\
A2 Alto & 0.80 & 2.23 & 0.69 & 0.50 & 1.60 & 0.11 & & 1.01 \\
A19 Alto & 1.83 & 6.22 & 0.86 & 0.54 & 3.41 & 0.00 & $* * *$ & 1.01 \\
\hline
\end{tabular}

Nota. AIC M1=320.41; AIC M2(Reducido)=291.07; *** $\mathrm{p}<0.001 ; * * \mathrm{p}<0.01 ; * \mathrm{p}<0.05 ;$ (.) $\mathrm{p}<0.1$ 
Los resultados indican que solo la conciencia social o la solidaridad (A19) aumenta la probabilidad de realizar emprendimientos sociales de manera significativa. Tal como se observa en la Tabla 2, en los estudiantes que tienen un alto grado de solidaridad autopercibida la probabilidad de emprender socialmente es 6.22 (odds ratio) veces mayor o, en otras palabras, la probabilidad de tener un emprendimiento social aumenta en un $86 \%($ Prob $=0.86)$.

\section{Conclusiones}

Los resultados empíricos muestran que existe un gran interés de parte de los estudiantes en las iniciativas empresariales con fines sociales, como lo evidencia el que la mayor parte de las empresas creadas para el concurso de emprendimiento de Ikasenpresa fueran de carácter social.

Por otra parte, el análisis desarrollado permite establecer ciertas diferencias entre los grupos de comparación. Los emprendedores potenciales se caracterizan así por poseer un mayor nivel autopercibido de capacidad innovadora, perseverancia, capacidad de identificar oportunidades, capacidad de trabajo en equipo, capacidad de aprendizaje, solidaridad y ética que quienes no emprenden. En cuanto al grupo de los potenciales emprendedores sociales, estos estudiantes presentan una mayor autopercepción en todos los atributos de personalidad y competencias propuestos -con excepción de la autoconfianza- en comparación con los potenciales emprendedores comerciales.

Ahora bien, a pesar de estas diferencias no se puede señalar que estadísticamente emprendedores potenciales y no emprendedores, así como también emprendedores potenciales sociales y emprendedores potenciales comerciales, constituyan grupos con perfiles claramente diferenciados. Por el contrario, se trata de grupos bastante homogéneos.

Sin perjuicio de lo anterior, el análisis de regresión reveló que existe una variable que aumenta significativamente la probabilidad de emprender socialmente, que es justamente uno de los factores que en mayor medida ha sido relevado como propio del emprendimiento social y la Economía Social: la solidaridad. En este sentido, se cumple parcialmente la hipótesis de este estudio, pues queda demostrado que los emprendedores sociales se asocian en mayor grado que los emprendedores comerciales con un atributo tal como la solidaridad, aunque no sucede lo mismo con la ética.

La aparente inconsistencia que puede observarse entre los resultados del análisis de medias y el modelo de regresión puede deberse a que las variables del estudio no responden a un modelo sumativo y, en conjunto, no aumentan la probabilidad de emprender, aunque lo puedan hacer de manera individual. De este modo, estos resultados sugieren la incorporación de variables y dimensiones distintas para futuras investigaciones, más que profundizar en el análisis de las mismas, puesto que las variables elegidas resultan ser insuficientes para explicar el fenómeno del emprendimiento potencial y del emprendimiento social. Lo anterior pasa por complementar la teoría de la personalidad con otros enfoques, por ejemplo, aquellos que prestan mayor atención a las variables del entorno en tanto determinantes de la intención emprendedora.

A pesar de sus limitaciones, la presente investigación ha sido una de las pocas que se ha propuesto estudiar atributos personales y su relación con intenciones de emprender en una amplia muestra de estudiantes en un campo poco explorado como es el de la formación profesional. Se espera que este estudio sirva de estímulo para futuras investigaciones similares que incluyan nuevas variables y prueben nuevas hipótesis, lo que permitirá eventualmente alimentar programas de formación de emprendedores en el ámbito escolar que visibilicen la Economía Social, ya que este tipo de empresas pareciera que responden a las necesidades prácticas y estratégicas de los adolescentes y jóvenes, e incluso se encuentran en mayor sintonía con los valores más próximos a la mentalidad propia de la edad como son las gestión democrática y responsable.

\section{Referencias bibliográficas}

Abir, S.A., Eyad, B.A. y Zahran, S.A. (2014) Factors impacting entrepreneurial intention: A literature review. International Journal of Economics and Management Ingeneering, Vol. 8, $\mathrm{N}^{\circ} 8$, pp. 1-4.

Acs, Z., Bosma, N. y Sternberg, R. (2011) The dynamics of entrepreneurship: Theory and evidence. Oxford: OUP.

Alonso, M.J. y Gálvez, C. (2008) El emprendedor y la empresa: Una revisión teórica de los determinantes a su constitución, Acciones e Investigaciones Sociales, $\mathrm{N}^{\circ} 26$, pp. 5-44.

Altinay, L., Madanoglu, M., Daniele, R. y Lashley, C. (2012) The influence of family tradition and psychological traits on entrepreneurial intention. International Journal of Hospitality Management, Vol. 31, $\mathrm{N}^{\circ}$ 2, pp. 489-499.

Alvord, S.H., Brown, L.D. y Letts, C.W. (2004) Social entrepreneurship and societal transformation: An exploratory study. The Journal of Applied Behavioral Science, Vol. 40, $\mathrm{N}^{\circ} \quad 3, \quad$ pp. 260-282. DOI: https://doi.org/10.1177/0021886304266847. 
Atxabal, A. (2014) Democracia y jóvenes, una aproximación desde las cooperativas. REVESCO. Revista de Estudios Cooperativos, Tercer Cuatrimestre, No 116, pp. 57-76. DOI: https://doi.org/10.5209/rev_REVE.2014.v116.45716.

Austin, J.E., Stevenson, H. y Wei-Skillern, J. (2006) Social and commercial entrepreneurship: The same, different or both? Entrepreneurship: Theory \& Practice, Vol. 30, № 1, pp. 1-22. DOI: https://doi.org/10.1111/j.15406520.2006.00107.x.

Bacq, S. y Janssen, F. (2011) The multiple faces of social entrepreneurship: A review of definitional issues based on geographical and thematic criteria. Entrepreneurship \& Regional Development, Vol. 23, No 5-6, pp. 373-403. DOI: https://doi.org/10.1080/08985626.2011.577242.

Baron, R. y Henry, R.A. (2010) Entrepreneurship: The genesis of organizations. En: Zedeck, S. (Ed.) APA handbook of industrial and organizational psychology (Vol. 1: Building and developing the organization). Washington DC: American Psychological Association, pp. 241-273.

Baum, J.R. y Locke, E.A. (2004) The relationship of entrepreneurial traits, skill, and motivation to subsequent venture growth. Journal of Applied Psychology, Vol. 89, $\mathrm{N}^{\circ}$ 4, pp. 587-598. DOI: https://doi.org/10.1037/00219010.89.4.587.

Bel Durán, P., Fernández, J., García, C., Lejarriaga, G. y Martin, S. (2016) Las sociedades cooperativas de enseñanza como impulsoras de iniciativas de creación de empresas. Madrid: UCETAM.

Borstein, D. (2004) How to change the world: Social entrepreneurs and the power of new ideas. Nueva York: Oxford University Press.

Brandstatter, H. (2011) Personality aspects of entrepreneurship: A look at five meta- analyses. Personality \& Individual Differences, Vol. 51, № 3, pp. 222-230. DOI: https://doi.org/10.1016/j.paid.2010.07.007.

Brinckerhoff, P. (2000) Social entrepreneurship: The art of mission-based venture development. Nueva York: John Wiley \& Sons.

Caballero, S., Fuchs, R.M. y Prialé, M.A. (2014) The influence of the Big 5 personality traits on the social enterprise start-up intentions: A Peruvian case. Taylor's Business Review, Vol. 4, No1, pp. 1-18. Recuperado desde: https://www.semanticscholar.org/paper/The-influence-of-personality-traits-on-social-the-SusyMar\%C3\%ADa/b479d81a27a008e03590de9f161d036ae013c025.

Comisión Europea (2016) Social enterprises and their eco-systems: Developments in Europe. Luxemburgo: Publications Office of the European Union. Recuperado desde: https://ec.europa.eu/social/main.jsp?catId=738\&langId=en\&pubId=7934\&furtherPubs=yes.

Crespo, N.F., Belchior, R. y Costa, E.B. (2018) Exploring individual differences in the relationship between entrepreneurial self-efficacy and intentions: Evidence from Angola. Journal of Small Business and Enterprise Development, Vol. 27, № 1. DOI: https://doi.org/10.1108/JSBED-03-2017-0105.

Cukier, W., Trenholm, S., Carl, D., y Gekas, G. (2011) Social entrepreneurship: A content analysis. Journal of Strategic Innovation and Sustainability, Vol. 7, $\mathrm{N}^{\mathrm{o}}$ 1, pp. 99-119. Recuperado desde: http://www.nabusinesspress.com/JSIS/CukierWeb.pdf.

Curto, M. (2012) Los emprendedores sociales: Innovación al servicio del cambio social. Cuadernos de la Cátedra la Caixa de Responsabilidad Social de la Empresa y Gobierno Corporativo, № 13. Recuperado desde: https://dds.cepal.org/redesoc/publication?id=2283.

Dacin, M.T., Dacin, P.A. y Tracey, P. (2011) Social entrepreneurship: A critique and future directions. Organization Science, Vol. 22, No5, pp. 1203-1213. DOI: https://doi.org/10.1287/orsc.1100.0620.

Darmanto, S. y Yuliari, G. (2018) Mediating role of entrepreneurial self efficacy in developing entrepreneurial behavior of entrepreneur students. Academy of Entrepreneurship Journal, Vol. 24, No 1, pp. 1-14.

Dart, R. (2004) The legitimacy of social enterprise. Nonprofit Management and Leadership, Vol. 14, No 4, pp. 11-424. DOI: https://doi.org/10.1002/nml.43.

Dees, J.G. (1998) The meaning of social entrepreneurship. Recuperado desde: https://centers.fuqua.duke.edu/case/wpcontent/uploads/sites/7/2015/03/Article_Dees_MeaningofSocialEntrepreneurship_2001.pdf.

Dees, J.G., Emerson, J. y Economy, P. (2001) Enterprising nonprofits: A toolkit for social entrepreneurs. Nueva York: John Wiley \& Sons.

Defourny, J., Hulgard, L. y Pestoff, V. (2014) Social enterprise and the Third Sector. Changing European landscapes in a comparative perspective. Londres y Nueva York: Routledge.

Defourny, J. y Nyssens, M. (2006) Defining social enterprise. En: Nyssens, M. (Ed.) Social enterprise: At the crossroads of market, public policies and civil society. Abingdon: Routledge, pp. 3-26.

Elkington, J. y Hartigan, P. (2008) The power of unreasonable people: How social entrepreneurs create markets that change the World. Boston MA: Harvard Business School Publishing.

Enciso, M., Gómez, L. y Mugarra, A. (2012) La iniciativa comunitaria en favor del emprendimiento social y su vinculación con la economía social: Una aproximación a su delimitación conceptual. CIRIEC-España, Revista de Economía Pública, Social y Cooperativa, №75, pp. 54-80. Recuperado desde: https://www.redalyc.org/articulo.oa?id=17425798004.

Ewen, R. (2010) An introduction to theories of personality. Sussex: Psychology Press.

Farber, V., Caballero, S., Prialé, M. y Fuchs, R.M. (2015) Social enterprises in Lima: Notions and operating models. Journal of Entrepreneurship \& Innovation in Emerging Economies, Vol. 1, No1, pp. 56-78. DOI: https://doi.org/10.1177/2393957514554986. 
Farrington, S.M. (2012) Does personality matter for same business success? South African Journal of Economic and Management Sciences, Vol. 15, No 4, pp. 382-401. DOI: https://doi.org/10.4102/sajems.v15i4.243.

Frank, H., Lueguer, M. y Korunka, C. (2007) The significance of personality in business start-up intentions, start-up realization and business success. Entrepreneurship \& Regional Development, Vol. 19, No 3, pp. $227-251$. https://doi.org/10.1080/08985620701218387.

Galera, G. y Borzaga, C. (2009) Social enterprise: An international overview of its conceptual evolution and legal implementation. Social Enterprise Journal, Vol. 5, $\mathrm{N}^{\mathrm{o}} \quad 3, \quad$ pp. $210-228 . \quad$ DOI: https://doi.org/10.1108/17508610911004313.

Gómez, J.M., Mira, I. y Martínez, J. (2007) Condicionantes de la actividad emprendedora e instituciones de apoyo desde el ámbito local: El caso de la provincia de Alicante. Revista de Empresa, № 20, pp. 20-31.

Guzmán, A. y Trujillo, M.A. (2008) Emprendimiento social: Revisión de literatura. Estudios Gerenciales, Vol. 24, N ${ }^{\circ}$ 109, pp. 105-125. DOI: https://doi.org/10.1016/S0123-5923(08)70055-X.

Harding, R. (2004) Social enterprise: The new economic engine? Business and Strategy Review, Vol. 15, № 4, pp. 3943. DOI: https://doi.org/10.1111/j.0955-6419.2004.00338.x.

Haugh, H. (2005) A research agenda for social entrepreneurship. Social Enterprise Journal, Vol. 1, No1, pp. 1-12. DOI: https://doi.org/10.1108/17508610580000703.

Hockerts, K. (2006) Entrepreneurial opportunity in social purpose business ventures. En: Mair, J., Robinson, J. y Hockerts, K. (Eds.) Social entrepreneurship. Basingstoke: Palgrave Macmillan, pp. 57-85.

Jiao, H. (2011) A conceptual model for social entrepreneurship directed toward social impact on society. Social Enterprise Journal, Vol. 7, № 2, pp. 130-149. DOI: https://doi.org/10.1108/17508611111156600.

Kerlin, J. (2006) Social enterprise in the United States and Europe: Understanding and learning from the difference. Voluntas, Vol. 17, No 3, pp. 247-263. DOI: https://doi.org/10.1007/s11266-006-9016-2.

Kirzner, I.M. (1997) Entrepreneurial discovery and the competitive market process: An Austrian approach. Journal of Economic Literature, $\mathrm{N}^{\mathrm{o}} 35$, pp. 60-85.

Kolvereid, L. e Isaksen, E. (2006) New business start-up and subsequent entry into self-employment. Journal of Business Venturing, Vol. 21, No 6, pp. 866-885. DOI: https://doi.org/10.1016/j.jbusvent.2005.06.008.

Kostetska, I. y Berezyak, I. (2014) Social entrepreneurship as an innovative solution mechanism of social problems of society. Management Theory and Studies for Rural Business and Infrastructure Development, Vol. 36. № 3 , pp. 569-577. DOI: https://doi.org/10.15544/mts.2014.053.

Kruse, P. (2020) Can there only be one? An empirical comparison of four models on social entrepreneurial intention formation. International Entrepreneurship and Management Journal, $\quad \mathrm{N}^{\mathrm{o}}$ 16, pp. 641-665. DOI: https://doi.org/10.1007/s11365-019-00608-2.

Lee, M., Battilana, J., y Wang, T. (2014) Building an infrastructure for empirical research on social enterprise: Challenges and opportunities. En: Short, J. (Ed.) Social entrepreneurship and research methods. Bingley: Emerald, pp. 241-264.

Lepoutre, J., Justo, R., Terjensen, S. y Bosma, N. (2011) Designing a global standardized methodology for measuring social entrepreneurship activity: The Global Entrepreneurship Monitor social entrepreneurship study. Small Business Economics, Vol. 40, No 3, pp. 693-714. DOI: https://doi.org/10.1007/s11187-011-9398-4.

Light, P.C. (2006) Reshaping social entrepreneurship. Stanford Social Innovation Review, Vol. 4, No 3, pp. $47-51$. Recuperado desde: https://wagner.nyu.edu/files/performance/ReshapingSE.pdf.

Mair, J. y Martí, I. (2006) Social entrepreneurship research: A source of explanation, prediction, and delight. Journal of World Business, Vol. 41, No 1, pp. 36-44. DOI: https://doi.org/10.1016/j.jwb.2005.09.002.

Mair, J. y Noboa, E. (2006) Social entrepreneurship: How intentions to create a social enterprise get formed. En: Mair, J., Robinson, J. y Hockerts, K. (Eds.) Social entrepreneurship. Basingstoke: Palgrave Macmillan, pp. 203-214.

Martin, R.L. y Osberg, S. (2007) Social entrepreneurship: The case for definition. Stanford Social Innovation Review, Vol. 5, $\quad \mathrm{N}^{\mathrm{o}} \quad 2, \quad \mathrm{pp}$. 28-39. Recuperado desde: $\underline{\text { https://www.law.berkeley.edu/php- }}$ programs/courses/fileDL.php?fID=7288.

Marulanda, F., Montoya, I. y Vélez. J. (2014) Aportes teóricos y empíricos al estudio del emprendedor. Cuadernos de Administración, Vol. $\quad 30, \quad \mathrm{~N}^{\mathrm{o}} \quad 51, \quad$ pp. 89-99. Recuperado desde: http://www.scielo.org.co/pdf/cuadm/v30n51/v30n51a10.pdf.

Ministerio de Educación, Cultura y Deporte (2015) La educación para el emprendimiento en el sistema educativo español. Año 2015. Madrid: Ministerio de Educación, Cultura y Deporte. Recuperado desde: https://www.selgipes.com/uploads/1/2/3/3/12332890/2016_-_ue_-_entrepreneurship_education_at_school_in_europe_sp.pdf.

Monzón, J.L. y Chaves, R. (2012) La Economía Social en la Unión Europea. Bruselas: Comité Económico y Social Europeo de la Unión Europea. Recuperado desde: https://www.eesc.europa.eu/resources/docs/qe-30-12-790-esc.pdf.

Monzón, J.L. y Chaves, R. (2017) Evolución reciente de la Economía Social en la Unión Europea. Bruselas: Comité Económico y Social Europeo de la Unión Europea. Recuperado desde: https://www.eesc.europa.eu/sites/default/files/files/qe-04-17-875-es-n.pdf.

Mor, S., Madan, S. y Chhikara, R. (2020) The risk-seeking propensity of Indian entrepreneurs: A study using GEM data. Strategic Change, Vol. 29, No 3 .

Murphy, P.J. y Coombes, S.M. (2009) A model of social entrepreneurial discover. Journal of Business Ethics, № 85, pp. 325-336. DOI: https://doi.org/10.1007/s10551-008-9921-y. 
Naa, J. y Shamuganathan, G. (2010) The influence of personality traits and demographic factors on social entrepreneurship start up intentions. Journal of Business Ethics, Vol. 95, No 2, pp. 259-282. DOI: https://doi.org/10.1007/s10551-009-0358-8.

Naushad, M. y Malik, S.A. (2018) The mediating effect of entrepreneurial self-efficacy in entrepreneurial intention-a study in Saudi Arabian context. Business Perspectives, Vol. 16, $\mathrm{N}^{\mathrm{o}}$ 1, pp. 267-275. DOI: http://dx.doi.org/10.21511/ppm.16(1).2018.26.

Newman, A., Obschonka, M., Schwarz, S., Cohen, M. y Nielsen, I. (2018) Entrepreneurial self-efficacy: A systematic review of the literature on its antecedents and outcomes, and an agenda for future research. Journal of Vocational Behavior, No 110-B, pp. 403-419. DOI: https://doi.org/10.1016/j.jvb.2018.05.012.

Nicholls, A. (2006) Playing the field: A new approach to the meaning of social entrepreneurship. Social Enterprise Journal, Vol. 2, No 1, pp. 1-5.

Nicholls, A. (2008) Social entrepreneurship: New models of sustainable social change. Oxford: Oxford University Press.

Nicholls, A. (2010) Institutionalizing social entrepreneurship in regulatory space: Reporting and disclosure by community interest companies. Accounting, Organizations and Society, Vol. 35, $\mathrm{N}^{\circ}$ 4, pp. 394-415. DOI: https://doi.org/10.1016/j.aos.2009.08.001.

Nicholls, A. y Cho, A.H. (2006) Social entrepreneurship: The structuration of a field. En: Nicholls, A. (Ed.) Social entrepreneurship. New models of sustainable change. Oxford: Oxford University Press, pp. 99-118.

Nissan, E., Castaño, M.S. y Carrasco, I. (2012) Drivers of non-profit activity: A cross-country analysis. Small Business Economics, Vol. 38, № 3, pp. 303-320. DOI: https://doi.org/10.1007/s11187-010-9276-5.

Nurdan, O. y Nancy, K.R. (2016) Entrepreneurial intention: Antecedents to entrepreneurial behavior in the U.S.A. and Turkey. Journal of Global Entrepreneurship Research, Vol. 6, N N 3. DOI: https://doi.org/10.1186/s40497-016$\underline{0047-\mathrm{x}}$.

Palamida, E. (2016) Determinants of entrepreneurial intentions: The interrelated role of background, situational and psychological factors. Tesis doctoral, Doctor of Philosophy, Newcastle University Business School, Inglaterra.

Peattie, K. y Morley, A. (2008) Social enterprises: Diversity and dynamics, contexts and contributions. Londres: Social Enterprise Coalition.

Pedrosa I. (2015) Evaluación de la personalidad emprendedora mediante un Test Adaptativo Informatizado. Tesis doctoral, Doctorado en Psicología, Universidad de Oviedo.

Peredo, A.M. y McLean, M. (2006) Social entrepreneurship: A critical review of the concept. Journal of World Business, Vol. 41, No 1, pp. 56-65. DOI: https://doi.org/10.1016/j.jwb.2005.10.007.

Pihie, Z.A.L. y Bagheri, A. (2018) Students' entrepreneurial regulation and intention to become an entrepreneur: A comparison between public and private universities. South African Journal of Business Management, Vol. 44, N ${ }^{\circ} 4$, pp. 25-32. DOI: https://doi.org/10.4102/sajbm.V44i4.166.

Rauch, A. y Frese, M. (2007a) Born to be an entrepreneur? Revisiting the personality approach to entrepreneurship. En: Baum, J.R., Frese, M. y Baron, R.J. (Eds.) The psychology of entrepreneurship. Mahwah NJ: Erlbaum, pp. 41-65.

Rauch, A. y Frese, M. (2007b) Let's put the person back into entrepreneurship research: A meta-analysis on the relationship between business owners' personality traits, business creation, and success. European Journal of Work and Organizational Psychology, Vol. 16, No 4, pp. 353-385. DOI: https://doi.org/10.1080/13594320701595438.

Reynolds, P.D., Bygrave, W.D. y Autio, E. (2004) Global Entrepreneurship Monitor 2003 executive report. Babson Park MA: Babson College.

Sánchez-García, J.C., Ward, A., Hernández, B. y Florez, J. (2017) Entrepreneurship education: State of art. Propósitos y Representaciones, Vol. 5, No 2, pp. 401-473. DOI: http://dx.doi.org/10.20511/pyr2017.v5n2.190.

Santos, F.M. (2012) A positive theory of social entrepreneurship. Journal of Business Ethics, Vol. 111, N 3, pp. 335351. DOI: https://doi.org/10.1007/s10551-012-1413-4.

Sassmannshausen, S.P. y Volkmann, C. (2018) The scientometrics of social entrepreneurship and its establishment as an academic field. Journal of Small Business Management, Vol. 56, $\mathrm{N}^{\mathrm{o}}$ 2, pp. 251-273. DOI: http://dx.doi.org/10.1111/jsbm.12254.

Seelos, C. y Mair, J. (2005) Social entrepreneurship: Creating new business models to serve the poor. Business Horizons, Vol. 48, N 3, pp. 241-246. DOI: https://doi.org/10.1016/j.bushor.2004.11.006.

Sesen, H. (2013) Personality or environment? A comprehensive study on the entrepreneurial intentions of university students. Education and Training, Vol. 55, No 7, pp. 624-640. DOI: https://doi.org/10.1108/ET-05-2012-0059.

Sharir, M. y Lerner, M. (2006) Gauging the success of social ventures initiated by individual social entrepreneurs. Journal of World Business, No41, pp. 6-20. DOI: https://doi.org/10.1016/j.jwb.2005.09.004.

Short, J.C., Moss, T.W. y Lumpkin, G.T. (2009) Research in social entrepreneurship: Past contributions and future opportunities. Strategic Entrepreneurship Journal, Vol. 3, No2, pp. 161-194. DOI: https://doi.org/10.1002/sej.69.

Singh, A (2016) The process of social value creation: A multiple-case study on social entrepreneurship in India. Nueva Delhi: Springer India.

Smith, G. (1999) Trait and process in personality theory: Defined within two contemporary research traditions. Scandinavian Journal of Psychology, Vol. 40, No4, pp. 269-276. DOI: https://doi.org/10.1111/1467-9450.404126.

Sullivan-Mort, G., Weerawardena, J. y Carnegie, K. (2002) Social entrepreneurship: Towards conceptualization and measurement. International Journal of Nonprofit and Voluntary Sector Marketing, Vol. 8, No 1, pp. 76-88. DOI: https://doi.org/10.1002/nvsm.202. 
Swarupa, S.G. y Guyal, R.K. (2020) Entrepreneurial intentions of students: Review of academic literature. International Journal of Scientific \& Engineering Research, Vol. 11, N $\mathrm{N}^{\mathrm{o}}$ 1, pp. 1146-1168. DOI: http://doi.org/10.14299/ijser.2020.01.02.

Tan, W.L., Williams, J. y Tan, T.M. (2005) Defining the 'social' in 'social entrepreneurship': Altruism and entrepreneurship. International Entrepreneurship and Management Journal, Vol. 1, No 3, pp. 353-365.

Thompson, J.L., Alvy, G. y Lees, A. (2000) Social entrepreneurship: A new look at the people and the potential. Management Decision, Vol. 38, No 5, pp. 328-338. DOI: https://doi.org/10.1108/00251740010340517.

Thompson, J.L. (2002) The world of the social entrepreneur. The International Journal of Public Sector Management, Vol. 15, No 5, pp. 412-431.

Tyszka, T., Cieslik, J., Domurat, A. y Macko, A. (2011) Motivation, self-efficacy, and risk attitudes among entrepreneurs during transition to a market economy. The Journal of Socio-Economics, $\mathrm{N}^{\circ}$ 40, pp. 124-131. DOI: https://doi.org/10.1016/j.socec.2011.01.011.

Unger, J.M., Rauch, A., Frese, M. y Rosenbusch, N. (2011) Human capital and entrepreneurial success: A metaanalytical review. Journal of Business Venturing, Vol. 26, $\mathrm{N}^{\mathrm{o}}$ 3, pp. 341-358. DOI: https://doi.org/10.1016/j.jbusvent.2009.09.004.

Uriarte, J. y González, P. (2007) Métodos e instrumentos de evaluación psicológica de jóvenes emprendedores. Recuperado desde: http://www.psicologiacientifica.com/metodos-e-instrumentos-de-evaluacion-psicologica-dejovenes-emprendedores/.

Valencia, J.A. y Marulanda, F.A. (2019) Evolución y tendencias investigativas en autoeficacia emprendedora: un análisis bibliométrico. Estudios Gerenciales, Vol. 35, No 151, pp. 219-232. DOI: https://doi.org/10.18046/j.estger.2019.151.3277.

Vernis, A. y Navarro, C. (2011) El concepto de ecosistema para el emprendimiento social. Revista Española del Tercer $\begin{array}{llllll}\text { Sector, } & N^{\circ} & \text { 17, } & \text { 67-84. } & \text { Recuperado desde: }\end{array}$ https://www.accioncontraelhambre.org/sites/default/files/documents/rets_17_0.pdf.

Volery, T., Muller, S., Oser, F., Naepflin, C. y Del Rey, N. (2013) The impact of entrepreneurship education on human capital at upper-secondary level. Journal of Small Business Management, Vol. 51, № 3, pp. 429-446. DOI: https://doi.org/10.1111/jsbm.12020.

Wang, J.H., Chang, C.C., Yao, S.N. y Liang, C. (2016) The contribution of self-efficacy to the relationship between personality traits and entrepreneurial intention. Higher Education, Vol. 72, $\mathrm{N}^{\mathrm{o}}$ 2, pp. 209-224. DOI: https://doi.org/10.1007/s10734-015-9946-y.

Wang, D., Wang, L. y Chen, L. (2017) Unlocking the influence of family business exposure on entrepreneurial intentions. International Entrepreneurship and Management Journal, Vol. 14, $\mathrm{N}^{\mathrm{o}}$ 4, pp. 951-974. DOI: https://doi.org/10.1007/s11365-017-0475-2.

Weerawardena, J. y Sullivan-Mort, G. (2006) Investigating social entrepreneurship: A multidimensional model. Journal of World Business, Vol. 41, No 1, pp. 21-35. DOI: https://doi.org/10.1016/j.jwb.2005.09.001.

Yim, J.W. y Weston, R. (2007) The characteristics of bioentrepreneurs in the Australian biotechnology industry: A pilot study. Journal of Management and Organization, Vol. 13, $\mathrm{N}^{\mathrm{o}} 4$, pp. 383-406. DOI: https://doi.org/10.1017/S1833367200003618.

Young, D. (2008) Alternative perspectives on social enterprise. En: Cordes, J.J. y Steuerle, E.C. Nonprofits and business. Washington DC: The Urban Institute Press.

Zahra, S.A., Gedojlovic, E., Neubaum, D.O. y Schulman, J.M. (2009) A typology of social entrepreneurs: Motives, search processes and ethical challenges. Journal of Business Venturing, Vol. 24, $\mathrm{N}^{\mathrm{a}}$ 5, pp. 519-532. DOI: https://doi.org/10.1016/j.jbusvent.2008.04.007.

Zhao, H. y Seibert, S.E. (2006) The Big Five personality dimensions and entrepreneurial status: A meta-analytical review. Journal of Applied Psychology, No91, pp. 259-271. DOI: https://doi.org/10.1037/0021-9010.91.2.259.

Zhao, H., Seibert, S.E. y Lumpkin, G.T. (2010) The relationship of personality to entrepreneurial intentions and performance: A meta-analytic review. Journal of Management, Vol. 36, $\mathrm{N}^{\mathrm{o}}$ 2, pp. 381-404. DOI: https://doi.org/10.1177/0149206309335187.

Zinga, A.C., Coelho, A.F. y Carvalho, F. (2013) Clustering of Angolan entrepreneurs: An analysis of their entrepreneurial posture. International Entrepreneurship and Management Journal, Vol. 9, № 4, pp. 483-500. DOI: https://doi.org/10.1007/s11365-011-0182-3. 